\title{
Evaluación de la digitalización frente a desastres natu- rales: Ios casos de Guatemala, Costa Rica y Nicaragua
}

\section{Ana Campos Hidalgo, María del Mar Barbero-Barrera}

Resumen: La frecuencia de eventos climáticos extremos, derivados del cambio climático, ha aumentado en las últimas décadas. Guatemala, Costa Rica y Nicaragua son tres de los países con mayor vulnerabilidad debido a la combinación de factores climáticos junto con los sociales y económicos. Aprovechando el impulso dado a la digitalización, en muchas áreas en desarrollo, para promover la comunicación y la accesibilidad a la información a todos los usuarios, la investigación que aquí se expone evalúa la efectividad y viabilidad de la introducción de aplicaciones digitales en los planes de gestión de riesgo en dichos países.

De acuerdo con los resultados de la investigación, se prevé que la comunicación a través de internet sea más efectiva y que, a pesar del potencial de las aplicaciones móviles, éstas podrán incluirse como recurso se acompañan de las políticas adecuadas y de los avances tecnológicos necesarios en cada país.

Palabras clave: Aplicaciones, desastres, cambio climático, tecnología, Costa Rica, Guatemala, Nicaragua.

Fecha de recepción: I de abril de 2020.

Fecha de admisión definitiva: 21 de julio de 2020. 
Assessment of Digitalisation in the Face of Natural Disasters: the Cases of Guatemala, Costa Rica and Nicaragua

Abstract: The frequency of extreme weather events caused by climate change has increased in recent decades. Guatemala, Costa Rica and Nicaragua are three of the most vulnerable countries owing to a combination of climatic, social and economic factors. The research presented here takes advantage of the impetus given to digitalization in many areas of development to promote communication and accessibility to information for all users, and evaluates the effectiveness and viability of the introduction of digital applications in risk management plans in these countries.

The results of the research indicate that Internet will be the most effective tool for communication and that, despite the potential of mobile applications, they are only an effective resource if accompanied by appropriate policies and technological advances in each country.

Key words: Applications, disasters, climate change, technology, Costa Rica, Guatemala, Nicaragua.

\section{Évaluation de la digitalisation face aux désastres naturelles: les cas du Guatemala, du Costa Rica y du Nicaragua}

Résumé: La fréquence des événements météorologiques extrêmes causés par le changement climatique a augmenté au cours des dernières décennies. Le Guatemala, le Costa Rica et le Nicaragua sont trois des pays les plus vulnérables en raison $d^{\prime}$ 'une combinaison de facteurs climatiques, sociaux et économiques. La recherche présentée ici profite de l'impulsion donnée à la digitalisation dans de nombreux domaines du développement pour promouvoir la communication et l'accessibilité à l'information pour tous les utilisateurs, et évalue l'efficacité et la viabilité de l'introduction d'applications mobiles dans les plans de gestion des risques dans ces pays.

Les résultats de ce travail indiquent qu'Internet sera l'outil de communication le plus efficace et que, malgré le potentiel des applications mobiles, celles-ci ne constituent une ressource efficace que si elles s'accompagnent de politiques appropriées et de progrès technologiques dans chaque pays.

Mots clé: Applications, désastres, changement climatique, technologie, Costa Rica, Guatemala, Nicaragua.

\section{Introducción}

En el año 2025, se alcanzarán 5.900 millones de suscriptores de telefonía móvil, según datos del Mobile World Congress (Mobile World Congress 2019), lo que implica que el $71 \%$ de la población mundial contará con dispositivo móvil. El elevado porcentaje de personas con acceso a internet pone de manifiesto el amplio potencial de aplicación de esta herramienta. De hecho, de acuerdo con el informe del Banco Mundial (2016), 7 de cada 10 hogares disponen de teléfonos móviles, porcentaje que es similar al de la población con acceso a agua segura para 
beber (71\%) (https://ourworldindata.org/water-access) y superior al del acceso a infraestructura de lavamanos (60\%) o al del acceso a saneamiento de gestión segura (40\%), entre otros (https://ourworldindata.org/sanitation).

Este hecho no solo influye en la cantidad de personas que puedan tener acceso a las nuevas tecnologías, sino que también genera un gran impacto en la economía mundial. La contribución de la telefonía móvil al Producto Interior Bruto mundial ha aumentado más del doble desde el año 2012 hasta alcanzar la cifra de 3,4 billones de euros (Global System for Mobile Communications). Los teléfonos inteligentes es un producto de mercado que ha visto incrementado su venta en esta última década. En el año 2014 se alcanzó la cifra de mil millones de smartphones vendidos y ha ido incrementando hasta conseguir en el año 2018 el número de 1.555 millones de ventas de este tipo de dispositivos (Statista).

Junto con la telefonía móvil, internet es otra de las grandes herramientas cuyo crecimiento ha sido exponencial en la última década. En la actualidad, el porcentaje de población con acceso a internet oscila entre el $95 \%$ en Norte América al $39 \%$ en África, siendo éste considerablemente inferior al 69\% de Hispanoamérica o el 54\% de Asia, según datos de diciembre de 2019 (https://www.internetworldstats.com/ stats. htm). Se espera que para el 2025, los suscriptores que utilicen el teléfono para acceder a internet lleguen a ser 5.000 millones frente a los 3.300 millones del año 2017 (Global System for Mobile Communications GSMA). El interés de este tipo de herramientas supone que la población pueda tener acceso a información de forma rápida y ágil e incluso garantizar una comunicación efectiva entre las entidades gubernamentales y la población. Internet a su vez está viviendo en los últimos años cambios que permiten constantes actualizaciones para provocar mejoras en su red. Es el caso de la tecnología $5 G$ que permitirá más capacidad de conexiones y más velocidad al poder navegar a 10 gigabytes por segundo (National Geographic). Se estima que para el año 2025 el $15 \%$ de las conexiones a internet se den por medio del $5 G$ (Global System for Mobile Communications). Gracias a este avance se podrán conectar más dispositivos y las alertas o la información llegará de una manera más rápida y directa.

Por otra parte, anualmente fallecen de media 60.000 personas al año como consecuencia de desastres naturales (https://ourworldindata.org/natural-disasters). Solamente en el 2018 se produjeron 315 fenómenos extremos. Causaron 11.804 muertes y 68 millones de afectados (Centre for Research on the Epidemiology of Disasters (CRED)). A pesar de que la cifra ha decrecido sustancialmente a lo largo del siglo XX y de lo que llevamos del siglo XXI, aún sigue llamando la atención dicha incidencia. Además de las pérdidas humanas, este tipo de situaciones deriva 
en sustanciales pérdidas económicas que, si se comparan con el Producto Interior Bruto de los distintos países del mundo, puede llegar a alcanzar el 0,5\% (https:// ourworldindata.org/natural-disasters). Desde 1980, los desastres naturales han causado pérdidas cercanas a 3 billones de dólares (Banco Mundial). Estos daños afectan directamente a la población más vulnerable. El cambio climático hará que aumenten considerablemente el número de desastres y para el 2030 se prevé 100 millones de personas se sumen a la situación de pobreza extrema (Informe Shok waves del Banco Mundial, 2019). A pesar de que dichos desastres no se pueden evitar, sus efectos sí podrían reducirse sustancialmente mediante planes específicos de gestión de riesgos que incorporaran medidas de planificación urbana y territorial junto con la incorporación de modelos predictivos (Williamson, 2002) incluso de movilidad de personas (Song et al). Para el año 2050 más de dos tercios de la población mundial habitará en las ciudades y la rápida urbanización creará ciudades no resilientes indefensas a los desastres naturales (Naciones Unidas), por lo que es necesario ahora más que nunca desarrollar protocolos de actuación. Dichos planes y modelos podrían, a su vez, articularse y vincularse a plataformas digitales ligadas al uso de telefonía móvil o de internet. En efecto, distintos estudios ponen de manifiesto la utilidad de herramientas digitales en fase de emergencia (Gething, 2011) o en la fase de postdesastre (Yang et al 2009; Zorn et al 2010). Sin embargo, existe escasa literatura sobre la adecuación de las áreas vulnerables a desastres naturales respecto a la disponibilidad de este tipo de dispositivos, por lo que, la investigación se centra en abordar este tipo de relaciones y evaluar qué posibles actuaciones de mejora pueden implementarse.

\section{Métodos}

La metodología seguida en la investigación se articula en tres fases, en la primera se analiza la vulnerabilidad de las distintas regiones frente a los desastres naturales, para, a continuación, evaluar el estado de digitalización de cada uno de los países y plantear propuestas de mejora para que el uso de la telefonía móvil como herramienta pueda emplearse de forma real en el plan de gestión de riesgos de cada uno de los países de estudio.

No obstante, dada la amplitud de la investigación, se decide acotar apoyándose en la investigación desarrollada por Armenteros (2017) sobre la vulnerabilidad frente a los efectos del cambio climático frente a desastres naturales. De acuerdo con dicha investigación Guatemala, Costa Rica y Nicaragua son tres de los países con mayor vulnerabilidad de todo el mundo. Esta circunstancia deriva no sólo de su emplazamiento: entre la Costa del pacífico y el Mar del Caribe, y de la afección 
de los movimientos tectónicos de las placas del pacífico, de Nazca y del Caribe, sino también por el denominado "corredor seco" (Martínez Valle, 2018) junto con las corrientes marinas y regímenes de viento de esta zona (Fernández, 2005). La vulnerabilidad natural de esta región se pone de manifiesto en los datos del número de desastres sufridos en las distintas regiones del mundo publicados por distintas entidades como el IDMC (Internal DisplacementMonitoring Center) oel Banco Mundial entre otros y recogidos por Armenteros en su investigación (2017). No obstante, un desastre natural no se convierte en catástrofe si el país o la región en la que incide tiene suficiente capacidad de actuación, es capaz de abordar su gestión de manera efectiva y eficaz y limitar sus consecuencias. Los países más empobrecidos son los más vulnerables frente a estas situaciones. Entre los años 1998 y 2018, el 91\% de muertes relacionadas con desastres en forma de tormentas se dieron en países de ingreso mediano y bajo, a pesar de que sólo el $32 \%$ de los fenómenos de tormentas se dieran en dichos países (Banco Mundial). En este sentido, otro importante parámetro a tener en consideración es el Índice de Desarrollo Humano (Human Development Index) que, desde el Programa de Naciones Unidas para el Desarrollo (PNUD) evalúa el nivel de desarrollo de un país. Este factor está asentado sobre tres pilares fundamentales: la esperanza de vida, la educación y el ingreso per cápita. Pues bien, de acuerdo con los datos de 20191, Guatemala y Nicaragua se encuentran en el puesto 126 con 0,651 de índice de desarrollo humano y con una esperanza de vida de 74,1 años. Frente a éstos, Costa Rica ocupa el puesto 68 de la lista mundial con 0,794 de IDH y 80,1 años de edad. Finalmente, es de gran interés la evaluación del IDH previsto, así como su crecimiento demográfico.

En el caso de Costa Rica el progreso del IDH ha experimentado una gran mejora desde 1990 hasta la actualidad al crecer un 0,69\% de media anual, superior al de otros países en Latinoamérica como México que tiene un 0,59\% de crecimiento (PNUD). En cuanto a la demografía, también va a sufrir un gran crecimiento. En el período entre 2017-2050 la población va a incrementarse en un 17,69\% y mayoritariamente será en contextos urbanos (Naciones Unidas). Guatemala, por otro lado, pertenece al grupo de países de desarrollo humano medio a pesar de tener un crecimiento anual del IDH del 1,11\% desde 1990 y haber subido dos puntos en la clasificación desde el año 2013 (PNUD). Sin embargo, sigue siendo un país con multitud de desigualdades. El $62 \%$ de la población vive en condiciones de pobreza extrema y aumenta en las zonas rurales e indígenas hasta alcanzar el $80 \%$. Estas diferencias no solo se dan ente etnias, también se dan por cuestiones de género (Naciones Unidas). Respecto al crecimiento demográfico, se estima que la población aumente en un $59,44 \%$ para el año 2050 e incluso alcanza el 89,31\%

'Más información en: http://hdr.undp.org/en/content/2019-human-development-index-ranking 
para el 2100 (Naciones Unidas). Nicaragua presenta un crecimiento anual del IDH del 0,99\% (PNUD).

Se han visto grandes avances en los últimos años como el aumento en 10 años de la esperanza de vida al nacer o el aumento en 4,5 años promedio de escolaridad. Sin embargo, estos valores todavía son inferiores a la media de los países Latinoamericanos (PNUD).

Para el año 2050 se prevé que la población crezca en un $26,66 \%$ y que también crezca la esperanza de vida hasta los 82 años (Naciones Unidas).

Una vez acotados los países objeto de la investigación, la primera parte de la investigación sobre la vulnerabilidad frente a desastres naturales se fundamenta en el análisis de los datos recogidos en diversas fuentes como el Banco Mundial o la Organización Mundial de la Salud. Extraeremos la situación específica de cada uno de los países y el tipo de desastre al que se enfrentan que, según lo indicado, se centran en terremotos, inundaciones, ciclones, sequías y deslizamientos.

Por otra parte, en aras a cruzar los datos anteriores con la adecuación de las herramientas digitales, analizaremos el estado de digitalización de cada uno de los países, basándonos en los datos recogidos de GSMA Intelligence, United States Census Bureau, Internet world stats y CIA world Facebook.

Finalmente, la investigación concluye con el análisis combinado de ambos factores y la propuesta de mejora para que los planes de gestión de riesgos de cada uno de los países puedan incorporar las aplicaciones digitales y las herramientas tecnológicas necesarias para mitigar los impactos de los futuros desastres naturales.

\section{Análisis de la situación frente a desastres naturales}

\section{I. Guatemala}

La vulnerabilidad de Guatemala se fundamenta no sólo en la ausencia de planificación urbanística o en su elevado índice de pobreza (Banco Mundial) sino también en el hecho de que, debido a su emplazamiento, este país está amenazado por distintos tipos de desastres: terremotos, inundaciones y tsunamis por su cercanía al mar, ciclones, incendios e incluso erupciones volcánicas. Su elevada densidad de población, la pobreza de sus habitantes y su situación geográfica le convierte en 
una región de alto riesgo (Fondo Mundial para la Reducción y Recuperación de Desastres). El Comité Permanente Interinstitucional de la Unión Europea situó en el año 2018 a Guatemala como el país latinoamericano con el mayor índice de riesgo, mayor vulnerabilidad y con menor capacidad de gestionar estos peligros (UNICEF).

Un estudio sobre desastres naturales en Guatemala elaborado para UNEPAR-UNICEF señala que entre los años 1530-1999, se han producido 21.509 eventos extremos, de los cuales la mayoría son fenómenos hidrometeorológicos (14.858). Uno de los eventos que más impacto negativo causó y sirvió para cambiar la estrategia a medio plazo fue el huracán Mitch en 1998. Causó 268 víctimas mortales, 280 heridos y 106.400 personas evacuadas (SEGEPLAN). Sin embargo, en la última década también se han producido grandes incidentes que son necesarios tener en cuenta. Según la Coordinadora Nacional para la Reducción de Desastres de Guatemala (CORNED), en 2016, se registraron 463 eventos que afectaron a un total de 445.628 personas. Solamente un año después (2017), la cifra ascendió a 2.187. El elevado número de eventos climáticos extremos provoca la pérdida de numerosas vidas y la generación de un elevado número de personas que se convierten en desplazados internos. Según el IDMC, en el año 2019, el número de desplazados internos en Guatemala derivados de desastres naturales ascendió a 21.000. Sin embargo, el número esperado de desplazamientos por año llegará a 42.763, de las cuales 30.125 serán a causa de terremotos, 12.578 por inundaciones, 50 por mareas de tormenta, 8 por tsunami, y 2 por viento ciclónico. Sin embargo, estos datos, lejos de ser anecdóticos señalan una tendencia que se viene repitiendo desde hace varios años. Desde el 2008, la cifra anual de desplazados debido a desastres naturales se mantiene en números altos, en ese año fueron 18.000. Dos años más tarde esa cifra subió hasta 163.000 ya que fue una fecha marcada por la erupción del volcán Pacaya y de la tormenta Ágatha. Fue el 2016 el año que contó con menos desplazados (1.700), pero en los últimos años se ha observado un pequeño repunte de estos datos (IDCM).

En el año 2017, se creó un documento (INFORM) para la gestión de riesgo ante desastres promovido principalmente por UNICEF. Su principal objetivo consistía en analizar el territorio de Guatemala para diseñar mejores estrategias a la hora de enfrentarse a futuras catástrofes. En este documento se crearon varios planos del territorio. Uno de ellos (figura 1) referencia el peligro y la exposición desde niveles de riesgo muy bajo hasta muy alto. En este plano se tuvieron en cuenta los riesgos humanos y naturales debido a la degradación ambiental y otros eventos naturales. El plano de índice de vulnerabilidad (figura 2) divide los territorios en cinco niveles de riesgo en base a aspectos socioeconómicos como desarrollo económico, población en dependencia, desigualdad y pobreza. Finalmente, el plano 
de índice de capacidad de respuesta (figura 3) mide esta capacidad frente a las instituciones e infraestructuras. Tiene en cuenta aspectos como la comunicación o acceso a sistemas de salud.

\section{Figura I. Mapa de índice de peligro y exposición de INFORM}

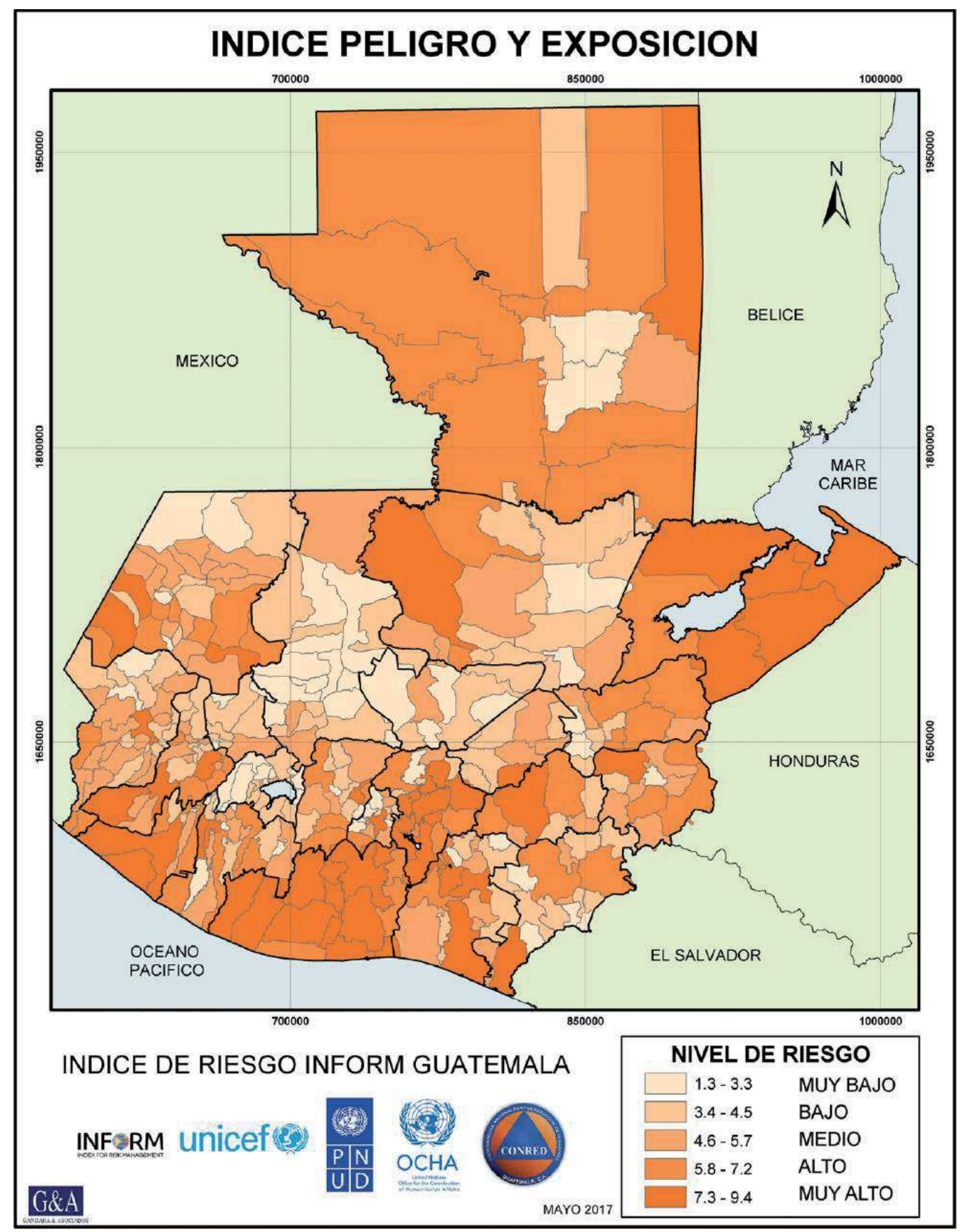

Fuente: CONRED. 
FIGURA 2. Mapa de índice de vulnerabilidad de INFORM

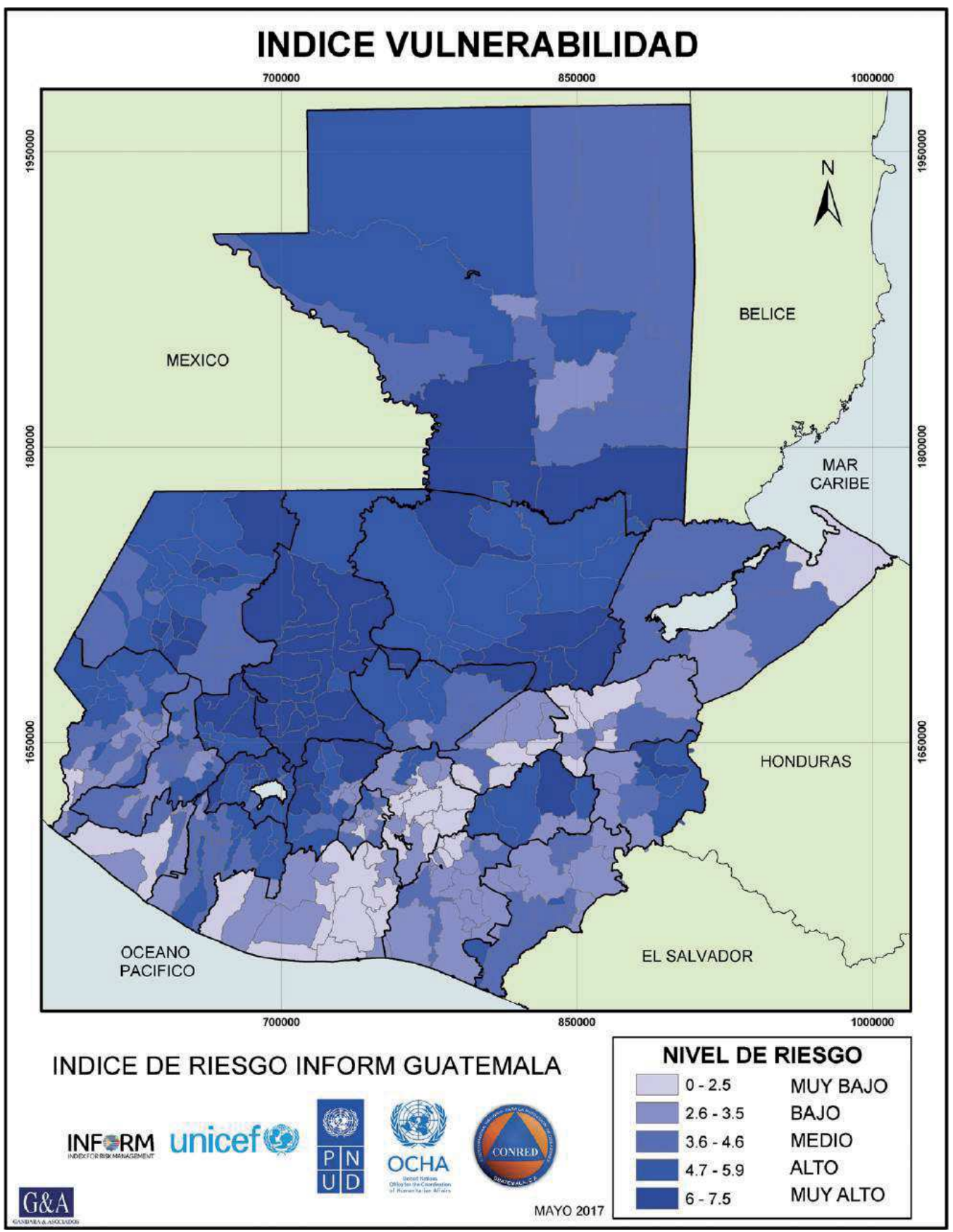

Fuente: CONRED. 


\section{FIGURA 3. Mapa de índice falta de capacidad de respuesta de INFORM}

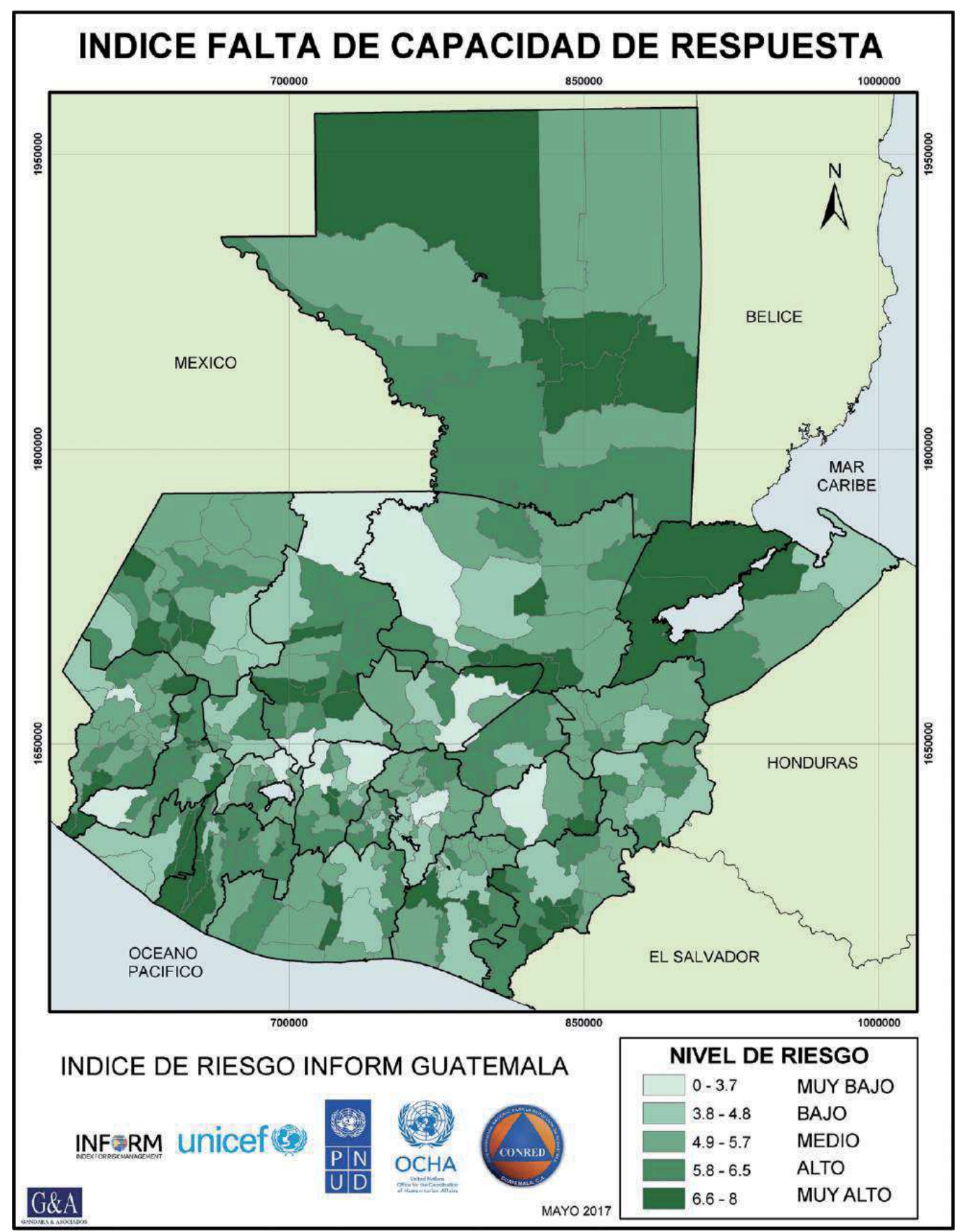

Fuente: CONRED.

Según el INFORM, la mayoría de los municipios con muy alto y alto riesgo en el índice de peligro y exposición son los municipios que tienen una gran densidad 
urbana, procesos de degradación medioambiental o que tienen una ubicación cercana a las principales rutas de comunicación. Estas localizaciones son las cabeceras departamentales de Guatemala, Retalhuleu, Quetzaltenango, Suchitepéquez (Mazatenango), Huehuetenango, Escuintla, Alta Verapaz (Cobán), Chimaltenango y Jalapa. En el estudio de vulnerabilidad, los municipios que más destacan negativamente se encuentran en los departamentos de Quiché, con 8 de sus 21 municipios y Sololá que tiene 7 de sus 19 municipios con el más alto grado de vulnerabilidad. Solamente 49 municipios de los 340 que tiene el país, cuentan con un grado de vulnerabilidad muy bajo y se encuentran localizados principalmente en el departamento de Guatemala, Escuintla, Zacapa y El Progreso. Finalmente, en la medición de la capacidad de respuesta se concluye que más del $85 \%$ del país está en riesgo por la falta de capacidad de respuesta, lo que afectaría negativamente a la mayoría de la población ante un desastre natural. Estos municipios de muy baja capacidad están distribuidos a lo largo de todo el territorio.

Tras la normativa internacional del Marco de Sendai para la reducción de desastres (2015), Guatemala comenzó a realizar grandes cambios en sus planes nacionales. La Coordinadora Nacional para la Reducción de Desastres (CONRED) desarrolla proyectos de respuesta ante eventos extremos y marca diferentes normativas a nivel de prevención, planificación y reconstrucción. La última modificación del Plan Nacional de Respuesta se ha realizado en el año 2019 (CONRED) y pretende definir de una manera clara las competencias interinstitucionales entre los distintos actores para reducir el posible impacto de los eventos extremos. Este país cuenta también con un Protocolo específico de lluvias en Guatemala para el año 2020 en el que se pretende definir la respuesta más adecuada ante este tipo de fenómeno y establecer la metodología de coordinación nacional. No es el único manual existente sobre un desastre en concreto. También se han desarrollado los protocolos en temporadas de incendio, de respuesta para atención de animales en un desastre y un protocolo de aplicación de excepciones de zonas declaradas en riesgo, todos ellos gestionados por CONRED. Sin embargo, en ninguno de ellos se redacta el papel fundamental que las nuevas tecnologías pueden ejercer en las respuestas ante estos incidentes.

\subsection{Nicaragua}

Nicaragua es un país que se sitúa en el "anillo de fuego" que rodea al Pacífico y une las placas tectónicas Coco y Caribe. Ha sido golpeado frecuentemente por terremotos, huracanes, erupciones volcánicas y sequías asociadas al fenómeno "El Niño" (OPS). Su latitud tropical causa que esté en el paso de grandes huraca- 
nes. Entre los años 1892-1998, el territorio ha sido afectado por 39 fenómenos meteorológicos graves originados en el Mar Caribe (OMS). Esto sumado a que nunca ha tenido una estrategia eficaz frente a tales amenazas hace que cada año muchas personas se vean afectadas (UNESCO).

Según un informe sobre el Inventario y Caracterización de Sistemas de Alerta Temprana en Nicaragua publicado por la UNESCO, este país es multiamenaza y ha sufrido a lo largo del tiempo eventos de diferente tipo. Sin embargo, se puede realizar una caracterización por zonas de estos desastres diferenciados por territorios. En la macro Región del Pacífico (departamentos de Chinandega, León, Managua, Masaya, Granada, Carazo y Rivas) se dan terremotos, tsunamis, inundaciones, sequías, erupciones volcánicas, deslizamientos y huracanes. En la macro Región Central (departamentos de Nueva Segovia, Madriz, Estelí, Jinotega, Matagalpa, Boaco, Chontales y Río San Juan) los principales ventos son inundaciones, sequías y deslizamientos. Finalmente, en la macro Región del Atlántico se suelen dar huracanes. El centro del país es recorrido por grandes cadenas montañosas que frenan la fuerza de los huracanes a su paso. Sin embargo, las lluvias que acompañan a estos fenómenos provocan graves inundaciones en zonas cercanas (OPS).

Entre los fenómenos acontecidos en los últimos años, según el Global Disaster Alert and Coordinations System, cabe destacar el Huracán Félix (2007) en el que fallecieron 159 personas, o el Huracán Mitch (1998) que dejó 3.200 fallecidos. Respecto a los terremotos, uno de los más destacados son el que se produjo en la ciudad de Managua (1931) que causó 1.500 muertes y 200 millones de dólares en daño. Esta misma ciudad sufrió otro sismo en el año 1972 y las víctimas mortales alcanzaron la cifra de 10.000. En definitiva, Nicaragua es uno de los cinco países de América Latina y el Caribe con mayor cantidad de pérdida de vidas humanas entre 1990 y 2011 por desastres naturales. Es también uno de los países que registran mayores cantidades de daños provocados por eventos hidrometeorológicos y climáticos (UNISDR). Estos incidentes no solo significan pérdidas humanas o daños económicos, sino que dejan cientos de desplazados internos cada año. Las personas desplazadas por eventos climatológicos en el año 2019 fueron 580 (IDMC).

En el 2000 se creó el Sistema Nacional para la Prevención, Mitigación y Atención a Desastres (SINAPRED). Su función es definir las políticas y planes de prevención ante desastres y aprobar el presupuesto para el Fondo Nacional para Desastres. Por otro lado, el Ministerio de Salud nombra puntos focales en los Sistemas Integral de Salud (SILAIS) y elabora planes de emergencia sanitarios locales y hospitalarios en estos casos (OPS). EI SINAPRED tiene un sistema con niveles de alerta para coordinar a los 
distintos agentes en función de la intensidad del fenómeno observado. La alerta verde se declara de forma preventiva tras identificar la presencia de un fenómeno natural. Esta alerta debe ser anunciada de manera pública a la población. La alerta amarilla se declara a partir del momento en que se evalúa el fenómeno identificado y suponga un peligro para el territorio. Implica la realización de acciones de preparación de acuerdo con el Plan de Respuesta Nacional. Por último, la alerta Roja se determina cuando se produce un fenómeno de forma súbita y se disponen todos los recursos que el Estado necesite para dar seguridad y salvaguardar a la población que se encuentre afectada o en situación de riesgo (SINAPRED).

\subsection{Costa Rica}

En el caso de Costa Rica, los desastres naturales de mayor frecuencia son principalmente los derivados de su condición de isla, esto es, inundaciones, terremotos, ciclones, aunque también erupciones volcánicas. Es un país ubicado sobre dos placas tectónicas muy activas, las placas de Cocos y Caribe. La altividad volcánica es el resultado del hundimiento de la plaza Cocos bajo la del Caribe (OPS). Además, esta región también está situada en el "cinturón de fuego" del Pacífico, lo que hace que y se vea influenciada por procesos naturales de gran impacto negativo. Esto hace que Costa Rica sea el quinto país más expuesto a desastres naturales según el Índice Mundial de Riesgo (Universidad de Naciones Unidas). Por otro lado, el gran crecimiento demográfico junto la densidad de poblacional, la urbanización no planificada, y las malas prácticas del uso de suelo aumenta el riesgo ante los desastres naturales.

En todo el país existen más de 200 focos volcánicos. Las regiones más vulnerables a la actividad volcánica son: El Valle Central y la Región Chorotega, en la que se encuentran los puntos más activos que además están en proximidad a núcleos de población (OPS). Pero no sólo es vulnerable a este tipo de eventos. Costa Rica sufre periódicamente los efectos de "El Niño", lo que crea graves sequías e inundaciones en algunas partes del territorio. Las zonas más afectadas son las del Sixaola en los meses de mayo y julio y la zona de Guanacaste en septiembre y octubre (OPS).

A ello se le añade, el IDH que, según se ha indicado anteriormente, tuvo en el año 2019 el valor de 0,794 y no ha dejado de crecer en los últimos años (PNUD). Por el contrario, el crecimiento demográfico es negativo ya que la tasa de fecundidad del país no ha dejado de descender desde los años 60 (Universidad de Costa Rica (CP-URC). El Centro Centroamericano de Población afirma que en el 2060 
comenzarán a morir más personas de las que nacen en el territorio nacional y el país está sufriendo continuamente un envejecimiento poblacional, algo que se debe tener en cuenta en las políticas sociales y los protocolos frente a desastres (CCP-URC).

De acuerdo con la Comisión Nacional de Emergencia (CNE), entre los desastres de mayor importancia acontecidos en las últimas décadas, cabe mencionar el Huracán Nate (2016) que dejó a 48 fallecidos y el Huracán Otto que sucedió ese mismo año y por el que fallecieron 10 personas (CNE). Pero esta isla ha sufrido terremotos (2009) en el que se perdió la vida de 25 personas, Tormenta tropical Alma (2008) con 11 fallecidos o un alud en Las Layas con 21 víctimas mortales (CNE). A parte de los fallecidos, estos eventos provocan grandes desplazamientos de personas que huyen de una zona en concreto para establecerse en otra parte del país. Según la IDMC, el número promedio de desplazados a lo largo del año 2019 fue de 390 aunque se espera que en los próximos años esta cifra ascienda a los 6.920, la mayoría provocados por terremotos.

En Costa Rica, la institución que se encarga de generar la normativa de actuación frente a desastres naturales es la Comisión Nacional de Prevención de Riesgos y Atención de Emergencia. Surgió en 1969 tras un período de crisis después de la erupción de los volcanes Iraza y Arenal y llevó a la creación de la Ley Nacional de Emergencia. La última reforma de esta ley se realizó en el año 2005 y tiene el objetivo principal de coordinar a los distintos agentes para salvaguardar la vida de los ciudadanos (Organización Panamericana de la Salud OPS). Esta comisión destina sus recursos en tres aspectos fundamentales: respuesta a las emergencias, rehabilitación de zonas afectadas y prevención. Trabaja por medio de varios comités como son el Comité Regional de Emergencia, Comité local de Emergencia, Comité Comunal de Emergencia y Comité de asesores técnicos (CNE). Esta comisión es la encargada de redactar los distintos manuales a seguir por las administraciones públicas y los distintos organismos de Costa Rica.

\section{Análisis de la tecnología}

De acuerdo con el objetivo de la investigación, en este apartado se evalúan los datos de telefonía e internet en cada uno de los países de estudio.

Las Figuras 4,5 y 6 resumen algunos de los datos identificados en los tres países objeto de estudio, aunque se analizarán en detalle a continuación, llama la aten- 
ción la diferencia entre Guatemala y Nicaragua respecto a Costa Rica, en la que la disponibilidad de telefonía móvil es notablemente superior pero también lo es el número de usuarios con internet.

\section{FIGURA 4. Resumen de los datos de accesibilidad a telefonía móvil, internet y redes sociales en Guatemala}

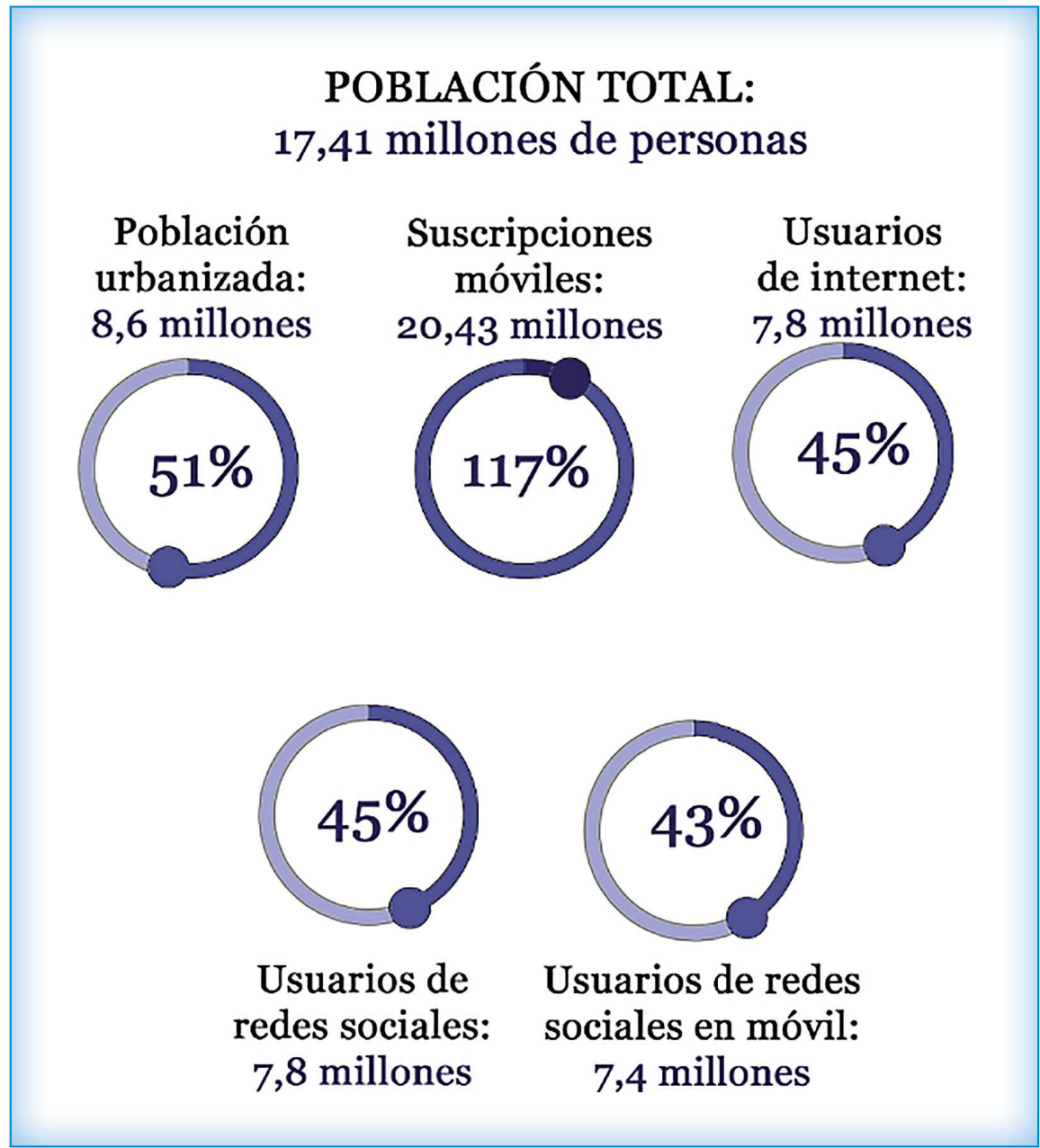

Elaboración propia a partir de GSMA Intelligence. 
FIGURA 5. Resumen de los datos de accesibilidad a telefonía móvil, internet y redes sociales en Nicaragua

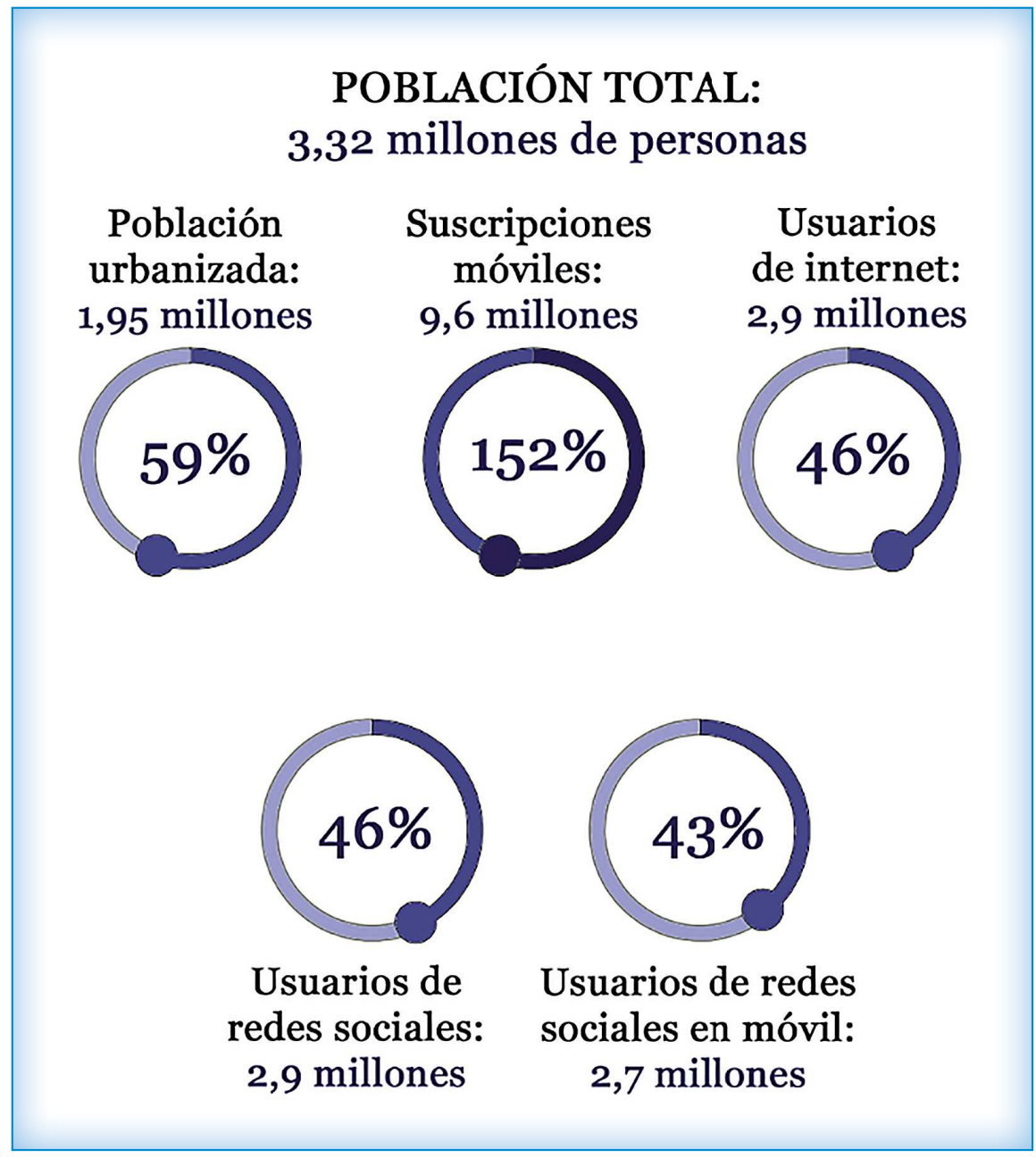

Elaboración propia a partir de GSMA Intelligence. 
FIGURA 6. Resumen de los datos de accesibilidad a telefonía móvil, internet y redes sociales en Costa Rica

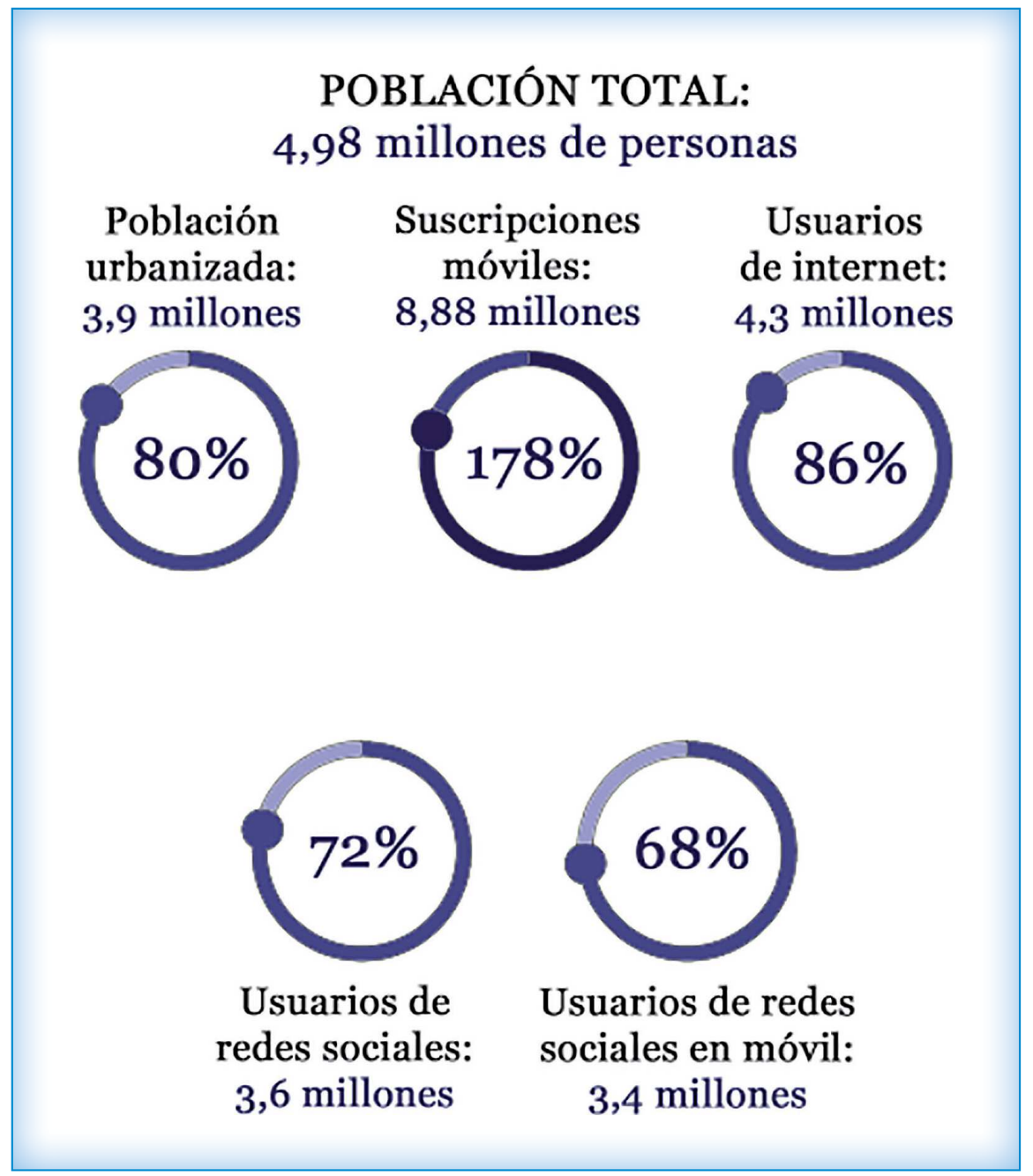

Elaboración propia a partir de GSMA Intelligence.

\section{I. Guatemala}

En Guatemala, el número de suscripciones móviles es muy elevado y asciende a 20,43 millones en una población de 17,25 millones de personas, lo que equivale a un $117 \%$ (GSMA Intelligence). Bien es cierto que dicho dato incluye teléfonos de empresas, oficinas, entes gubernamentales o incluso asociaciones, por lo tanto, no implica que toda la población disponga de un teléfono móvil, aunque sí un elevado porcentaje. Este dato es corroborado por la Agencia de los Estados Unidos para el Desarrollo Internacional (USAID) según la cual la mayoría de los hogares dis- 
ponen de telefonía móvil con objeto de mantenerse en contacto con sus familiares que han emigrado (USAID). De hecho, según se indica en el propio documento:

Casi todas las familias de aquí tienen un integrante en los Estados Unidos; de allá viene el recurso, viene el teléfono, y si no viene el teléfono viene el dinero y hay personas que tal vez nunca han tenido la posibilidad de comprarse algo, tienen dinero, empiezan a comprarse de todo y allí vienen los celulares.

No obstante, de esta afirmación general, es preciso destacar que tal y como reconoce el informe, las familias más pobres y aquéllas que residen en entornos muy rurales no cuentan con ningún dispositivo móvil en la familia. El $49 \%$ de los ciudadanos vive en territorios rurales y en la mitad de estos municipios, más del $75 \%$ de sus habitantes vive en situación de pobreza (INE). Los departamentos más afectados por este problema son Alta Verapaz, Sololá, Totonicapán y Suchitepéquez (Banco Mundial). Estas personas no sólo sufren las consecuencias económicas, sino que también son vulnerables a factores educativos, aislamiento geográfico e impactos de desastres naturales (Banco Mundial).

Frente a la tenencia de telefonía móvil, su conexión a internet es limitada y tan sólo 7,4 millones de personas tienen conexión a internet en sus teléfonos (GSMA Intelligence), aunque este dato es algo inferior a la disponibilidad de internet que se cifra en 7,8 millones de usuarios (Internet World stats). Esta cantidad es la misma que las personas que usan redes sociales en su día a día (CIA world facebook). En este sentido, cabe destacar que 8,6 personas residen en áreas urbanizadas (51\% de la población) lo que puede derivar en la falta de accesibilidad a dicha infraestructura en las localidades rurales, que será preciso tener en consideración en aras de proponer una tecnología accesible a la totalidad de la población.

Un dato interesante es el rango de población que utiliza internet, siendo éstos principalmente hombres de entre 18 y 35 años (USAID), lo que es de gran interés debido a la apertura tecnológica de estas generaciones y al potencial de uso de la tecnología para prevenir y limitar los efectos de los desastres naturales. También es interesante destacar que las personas de más edad (mayores de 35), que sólo suponen un $25,2 \%$ de población según datos del INE en el año 2014, rehúsan utilizar este dispositivo ya que consideran que es difícil aprender a utilizarlos y solamente adquieren uno para llamadas o SMS (USAID) lo que pone de manifiesto la importancia de que los planes no sólo contemplen aplicaciones digitales asociadas a móviles sino que dicha información pueda transmitirse también por SMS o por otros medios que garanticen dar cobertura a mayor número de población. Sin embargo, Guatemala tiene una estructura de población joven y con perspectivas de crecimiento. La mayor proporción de nacimientos se da en madres 
entre 20 y 24 años $(29,8 \%)$, seguido de nacimientos en madres de 25 a 29 años $(22,8 \%)$ y de mujeres entre 15 y 19 años $(19,4 \%$ ) (INE Guatemala). Por lo tanto, las familias con padres mayores a 40 años tienen muchas posibilidades de tener hijos familiarizados con la tecnología y que puedan utilizar los dispositivos móviles para mejorar su calidad de vida.

\subsection{Nicaragua}

En Nicaragua el número de suscripciones móviles asciende a 9,6 millones (152\% de la población), lo que pone de manifiesto la amplia cobertura de esta tecnología entre la ciudadanía (GSMA Intelligence, United States Census Bureau). Este valor es ligeramente superior al que habíamos indicado en el caso de Guatemala también porque su población urbana es algo superior, siendo en este caso de 1,29 millones de personas lo que supone un $59 \%$ de la población.

A pesar del elevado número de suscripciones móviles, en el año 2017, la Cámara de Nicaragua de Internet y Comunicaciones (CANITEL) destacaba la dificultad de su adquisición por parte de la población debido a su elevado coste. Lo que también es uno de los limitantes de la conexión a internet, con tan sólo 2,9 millones de usuarios. Esta cifra desciende si hablamos de usuarios con redes sociales en el móvil, que son 2,7 millones (Internetworld stats, CIA world factbook). Esta cifra indica que sólo el $46 \%$ de la población del país dispone de estas herramientas, aunque es previsible que aumente tal y como ha sucedido en los últimos años, en los que las conexiones $4 G$ han aumentado un $90 \%$ y las de banda ancha un $4,5 \%$ (CANITEL).

Por otra parte, el hecho de que la población urbanizada, 1,95 millones, sea ligeramente superior que la de Guatemala también justifica al igual que las suscripciones móviles. Sin embargo, más de la mitad de los ciudadanos no cuenta todavía con acceso a internet ni a los servicios que éste permite como las redes sociales. Nos encontramos en otra situación en la que la mayoría de la población no puede usar aplicaciones móviles frente a un desastre natural y es algo que hay que tener en cuenta a la hora de proyectar planes de gestión de catástrofes.

En cuanto a la conexión a internet, sin embargo, es destacable el dato de que, en el año 2017, 29 municipios con menos de 50.000 habitantes carecían de cobertura, de los cuales el $90 \%$ presentaban niveles de pobreza extrema o alta (CANITEL, 2017). Desde nuestro punto de vista este dato destaca la necesidad de acometer planes de accesibilidad digital para la población más vulnerable para acercar 
la información a esta población, pero además, de garantizar una comunicación efectiva y rápida en caso de desastres naturales.

\subsection{Costa Rica}

Finalmente, Costa Rica, el número de suscripciones móviles es de 8,88 millones, es decir, un $178 \%$ de la población total (4,98 millones), lo que supone el mayor número de suscripciones de los tres casos de estudio (GSMA Intelligence, United States Census Bureau), por lo tanto, con un gran potencial en el uso de la telefonía móvil como recurso para comunicar a la población alertas o recomendaciones de forma ágil.

Una vez más, aunque existe una amplia difusión de telefonía móvil, el número de usuarios de internet se limita al $86 \%$ con 4,3 millones de personas (Internetworld stats). Aunque, según el Centro Internacional de Política Económica, los usuarios lo identifican como indispensable en su vida con una frecuencia de uso de entre 1-5 horas/día. En cuanto al uso de internet, el 78,3\% utiliza este medio para mantenerse al tanto de las noticias internacionales y para ver posibles sucesos que afecten a su población (Centro Internacional de Política Económica). Por otra parte, es destacable que casi la mitad de propietarios de un teléfono móvil tienen una tarifa de pospago sin internet. Un 38,7\% cuentan con pospago con internet, $29,3 \%$ prepago sin internet y el $8,5 \%$ restante tiene móvil con prepago con internet. Estos datos reflejan que hay un gran porcentaje de personas que solamente pueden acceder a internet con una red wifi en sus proximidades. No obstante, entre los problemas reportados destacan la baja velocidad de conexión y la frecuencia de caídas.

Al igual que internet, el uso de redes sociales está muy extendido entre la población, identificando 3,6 millones de usuarios (72\% de la población), aunque sólo un $68 \%$ (3,4 millones de personas) lo tienen asociado a su teléfono móvil.

Finalmente, en cuanto al rango de edad de los usuarios, la población más joven está más familiarizada con su uso. En torno al $54 \%$ son personas de entre 18 y 40 años y el $45 \%$ tiene más de 40 (CINPE). Por lo tanto, las nuevas generaciones tienen más herramientas para recibir alertas de desastres por medio del móvil. 


\section{Propuestas de mejora}

Correlacionando los datos de desastres naturales con los de disponibilidad de telefonía móvil y de accesibilidad a internet y redes sociales. A continuación, se plantean distintas propuestas de mejora para cada uno de los países objeto de investigación.

\section{I. Guatemala}

Como se ha analizado en el apartado anterior, la disponibilidad de telefonía móvil es limitada a un cierto porcentaje de población siendo, por lo tanto, preciso que los sistemas de aviso y alerta sean diversos, vía no sólo de aplicaciones móviles sino también SMS, radioy televisión, entre otros. En estos casos, la programación de alertas a distintos niveles puede ser una solución efectiva para garantizar el conocimiento de la población, esto es, capacitando a un líder de la comunidad que sea el que garantice la transmisión de avisos, y garantizando un buen sistema de coordinación que sea eficaz en la transmisión de mensaje. Este sistema se aprovecharía además de la percepción positiva de este tipo de canales de comunicación por parte de la sociedad (Western Highlands Integrated Program WHIP 2017), siempre y cuando resulte gratuito pudiéndolo incorporar al Plan Nacional de Respuesta elaborado por la plataforma de Coordinación Nacional para la Reducción de desastres. En este plan se explica claramente los sistemas de alerta y los distintos niveles, pero en ningún momento menciona la utilización de canales digitales para alertar a la población. Bien es cierto, que el gobierno ya está utilizando mensajes de SMS y radio para distintas iniciativas de crecimiento económico, de salud y de educación tales como las incorporadas en el programa Western Highlands Integrated Program (WHIP), por lo que sería coherente aprovechar la infraestructura para incorporar e los sistemas de avisos aquellos referentes a catástrofes naturales, pudiéndose dar alertas sobre desastres próximos y consejos de actuación.

Las diferentes investigaciones ponen de manifiesto la importancia de capacitar a la población y de sensibilizarla frente a este tipo de programas (Homier et al 2018) de tal forma que conozcan cómo actuar de una forma efectiva y sensibilizarles evitando desconfianza sobre la privacidad y seguridad de los números telefónicos. De hecho, la necesidad de formación digital se pone de manifiesto en este testimonio recogido por la USAID en un grupo focal de hombres mayores de Tocache:

Yo me he dado cuenta en otras personas que tienen un estudio más avanzado. [El celular] es para ellos como una herramienta muy positiva. Pero en el caso de nosotros, que me den a mí un celular que es muy avanzado, que traiga todo, digamos, la tecnología 
que estamos... no se puede tener acceso a ellos porque no puedo porque me falta una educación. (USAID, 2016)

A tal respecto, es fundamental que exista una inversión en educación tecnológica no sólo en las escuelas, dirigida a los niños y adolescentes, sino también en el ámbito de las personas adultas utilizando los medios de comunicación, sobre todo la radio, ya que es el sistema más utilizado por esta parte de la población.

Para finalizar, como se ha puesto de manifiesto en la sección anterior, un elevado número de familias no tienen disponibilidad económica para tener acceso a un teléfono móvil. En este sentido, el gobierno debería facilitar al menos uno por comunidad, así como también dispositivos para acceso a internet de tal forma que se evite la exclusión social derivada del acceso a tecnología de estos ciudadanos que, además, son los más vulnerables.

\subsection{Nicaragua}

En este caso, la suscripción a telefonía móvil asciende al 152\% por lo que sí se dispone de un elevado número de teléfonos que facilitan las actuaciones en esta materia. Sin embargo, uno de los problemas principales de este país es que la red de conexión es insuficiente ralentizando la velocidad de internet y dificultando su uso como herramienta de comunicación. En este sentido, la mejora de la red de conexión, quizá mediante inversiones público-privadas, es uno de los primeros pasos a abordar, dando cobertura, en primer lugar, a los municipios que carecen de ella. Al igual que se indicó en Guatemala, la realización de campañas de educación y concienciación tecnológica para estas comunidades rurales es un aspecto clave para sacar el máximo aprovechamiento de esta tecnología.

Los planes de mitigación de riesgos se organizan en los niveles de alerta ya mencionados. Se prevé la utilización de radio y medios de comunicación tradicionales. Sin embargo, no mencionan la incorporación de herramientas como las aplicaciones móviles o los SMS para llegar a la máxima ciudadanía posible.

Finalmente, al igual que en Nicaragua, debería facilitarse el acceso a telefonía móvil e internet por parte de la población más vulnerable. En el caso del primero, en primera instancia dando cobertura a través de los líderes de la comunidad de tal forma que permita una comunicación rápida y directa con las entidades gubernamentales. En el caso de internet, con objeto de evitar la exclusión social y reducir el analfabetismo tecnológico que permita a los usuarios conocer de primera 
mano información de diversa índole, desde oferta de puestos de trabajo hasta el acceso al conocimiento y a bibliografía disponible en internet en la actualidad.

\subsection{Costa Rica}

Como se ha indicado en el apartado anterior, Costa Rica presenta una situación completamente diferente a las anteriormente analizadas. Con un porcentaje de suscripciones a telefonía móvil del 178\%, la población emplea este tipo de dispositivos de forma habitual, para mantenerse al tanto de las noticias internacionales, lo que implica que, en este caso, sí, el uso de aplicaciones digitales coordinadas por las entidades de salvamento podría ser de gran utilidad para la reducción de las consecuencias de los desastres naturales.

Al mismo tiempo, dado que uno de los problemas que más denuncian los usuarios es la caída de la red de conexión durante horas o incluso día, es imprescindible la realización de mejoras en la infraestructura para garantizar la cobertura y reducir el riesgo de fallos en caso de desastre natural. Se debería manejar la posibilidad de internet con satélite para todas aquellas zonas rurales o alejadas a las que no llega la conexión por cable.

A pesar de que la Comisión Nacional de Prevención de Riesgos y Atención de Emergencia cambia los manuales de actuación periódicamente, todavía no han incluido las nuevas herramientas tecnológicas dentro del sistema de alerta. Por lo tanto, se debería realizar una campaña de publicidad y talleres de concienciación sobre este tipo de aplicaciones, especialmente de aquellas gestionadas por el gobierno central para que sirva como recurso en la gestión de riesgos ante desastres.

Finalmente, la mayoría de las familias en Costa Rica cuentan con un dispositivo móvil. Sin embargo, al igual que ocurre en los dos países estudiados, la población rural todavía no cuenta con suficientes teléfonos ni con una infraestructura que asegure conexión en caso necesario. Será necesario establecer una serie de medidas sociales y ayudas económicas que apoyen a este sector de la población para que al menos los líderes comunitarios tengan este recurso y alerten al resto de ciudadanos. 


\section{Conclusiones}

Anualmente fallecen alrededor de 60.000 personas (Center for Rerearch on the Epidemiology of Disaster) como consecuencia de desastres naturales, siendo previsible que dicha cantidad aumente como consecuencia de los fenómenos asociados al cambio climático en ciertas áreas. Sin embargo, el uso extensivo de tecnología móvil podría mitigar los efectos alertando a la población con suficiente antelación para que se puedan adoptar medidas reductoras, así como facilitando la comunicación y la coordinación entre distintos servicios de emergencia. En efecto, en el mundo, el número de suscripciones a telefonía móvil asciende al $71 \%$ de la población mundial, porcentaje que, llamativamente, es superior a la tasa de población con acceso a saneamiento de gestión segura (40\%) e igual al porcentaje que tiene acceso a agua segura para beber (71\%). Sin embargo, estos datos son globales y, por lo tanto, es preciso realizar un análisis pormenorizado país por país y región por región para evaluar la adecuación de esta tecnología a la realidad social de cada área.

A partir de una investigación previa desarrollada por Armenteros (2017) se seleccionan tres países de estudio: Guatemala, Nicaragua y Costa Rica, debido a su elevada susceptibilidad a sufrir catástrofes naturales, como consecuencia de la combinación del riesgo de desastres naturales, derivados de su emplazamiento, junto con la debilidad del sistema público que, con un IDH bajo y una tasa de crecimiento elevada podría derivar en la falta de capacidad del estado de abordar este tipo de situaciones.

En cada uno de estos países se analiza, por una parte, el tipo de desastres naturales que podrían acontecer y la disponibilidad de planes de gestión de riesgos y lo que éstos comprenden, $y$, por otra parte, la accesibilidad tecnológica. Del estudio se desprende, en primer lugar, que las aplicaciones digitales todavía no se han incorporado como medio de comunicación ante desastres naturales por parte de las entidades gubernamentales. Los gobiernos podrían utilizar este canal para alertar a la población al igual que ya sucede con la radio o televisión. Sin embargo, para que esto fuese posible, las aplicaciones no deben ser gestionadas por entidades privadas sino que tiene que existir un compromiso de las administraciones públicas para este fin, empleándolas también como herramientas de predicción o incluso de coordinación entre distintas entidades, de tal forma que, a partir de la aplicación pudiera darse información a la población de diversa índole, no sólo avisos y recomendaciones sino también información sobre la ubicación de albergues, el emplazamiento de centros de emergencias sanitarias, la localización de fuentes de agua potable segura, etc. 
Por otra parte, la carencia de infraestructuras necesarias para que el uso de esta tecnología llegue a toda la población con sistemas de conexión de red lentas o que se caen con frecuencia. Al respecto, en primer lugar, sería preciso promover inversiones público-privadas para mejorar la cobertura en la totalidad del territorio nacional, optando, en los casos más remotos por la conexión vía satélite. Asimismo, y mientras que se mantengan estas condiciones, el sistema de alertas debería ampliarse a otros medios como SMS, radio o televisión.

Por otra parte, se observan notables diferencias en cuanto a la digitalización de los países de tal forma que, mientras que en Costa Rica la población está habituada al uso de la tecnología móvil para comunicarse o estar el tanto de la información. No sucede así en Guatemala y en Nicaragua donde la tasa de digitalización es reducida y la población pobre queda excluida de ella. En este sentido, llama la atención especialmente el caso de Guatemala donde la mitad de la población reside en áreas rurales y la mayor parte de la población no tiene acceso a internet. En este caso, la implantación de sistemas de alerta o de aviso a través de aplicaciones digitales no sería de utilidad si previamente no se resuelven los problemas de falta de acceso a la tecnología o el analfabetismo digital. En este caso, se deben centrar los esfuerzos en buscar recursos para que al menos los líderes de las comunidades y personas de referencia puedan utilizar las herramientas tecnológicas como sistema de alerta y que lo traslade a sus conciudadanos. Junto con esto se observa la necesidad de acometer programas de educación tecnológica que no sólo permita a los ciudadanos conocer cómo utilizarlo sino que también sirva para reducir el riesgo de exclusión social y el analfabetismo tecnológico.

En definitiva, las aplicaciones digitales se pueden convertir en un recurso que las autoridades gubernamentales pueden usar como método de comunicación y alerta con la ciudadanía. Sin embargo, cada país presenta un contexto diverso y características propias que deben tenerse en cuenta. Es importante conocer el alfabetismo tecnológico que existe en cada área y región del país y el conocimiento y uso que los ciudadanos hacen de internet para que las soluciones a cada caso sean particularizadas y adecuadas a sus necesidades y vulnerabilidades específicas, de tal forma que las medidas que se implementen sean eficaces y extensivas al mayor número de población y permitan salvar vidas y mitigar el impacto de los desastres naturales futuros. 


\section{Bibliografía}

BANCO MUNDIAL (2016). "Shock Waves: Managing the impacts of climate change on poverty". DC 20433. https://www.bancomundial.org/

Calvo-Solano, O. D., Quesada-Hernández, L., Hidalgo, H. y Gotlleb, Y. (2018). "Impactos de las sequías en el sector agropecuario del Corredor Seco Centroamericano". Scielo, 29, p.3. DOI: 10.15517/ma.v29i3.30828

CÁmARa NicaragüEnSE de Internety COMunicaciones (CANITEL): hHtps://canitel.org.ni/

Center for Research on the Epidemiology of Disaster (CRED): hittps://www.cred.be/

Centro Centroamericano de Población de la Universidad de Costa Rica (CCP-URC): https://ccp.ucr.ac.cr/

Centro Internacional de Política Económica (CINPE): hHtp://www.cinpe.una.ac.cr/ CIA WORLD FACEBOOK: https://www.cia.gov/library/publications/ the-world-factbook/

Comisión Nacional de Prevención de Riesgos y Atención de Emergencias de Costa Rica (CNE): https://www.cne.go.cr/

Coordinadora Nacional para la Reducción de Desastres de Guatemala (CORNED): https://conred.gob.gt/site/index.php

FernándeZ, A. (2005). Comarcas vulnerables: riesgos y desastres naturales en Centroamérica y el Caribe. CRIES ed. ISSN: 1016-9628

GetHing, P. W. y TAtem, A. J. (2011). Can mobile phone data improve emergency response to natural disasters? PLoS Medicine, 8, e1001085

Global System for Mobile Communications: https://www.gsma.com/

Grupo Focal de Hombres Mayores de Tocache (2016). Estudio sobre telefonía celular, USAID, p. 25

Homier, V., Hamad, R., Larocque, J., Chassé, P., Khall, E. y Franc, J. M. (2018). "A randomized trial comparing telephone tree, text messaging, and instant messaging 
app for emergency department staff recall for disaster response". Prehospital and disaster medicine, 33, 471-477. DOI: 10.1017/S1049023X18000912

International Displacement Monitoring Center: https://www.internal-displacement. org/

INTERNAL WORLD STATS: https://www.internetworldstats.com/stats.htm

Kryvasheyeu, Yl., Chen, H., Obradovich, N., Moro, E., Van Hentenryck, P., Fowler, J. y CEBRIÁN, M. (2016). Rapid assessment of disaster damage using social media activity. Science Advances, 2 (3), e150079

Martínez-Valle, A. (2018). "Perspectivas de la adaptación al cambio climático en el corredor seco Centroamericano". CGSpace. Disponible en: https://cgspace. cgiar.org/handle/10568/100528

MOBILE WORLD CONGRESS: hHtps://www.mwcbarcelona.com/

NACIONES UnidAS PARA EL DeSARROLLO (PNUD): https://www.undp.org/content/undp/ es/home.html

National Geographic: https://www.nationalgeographic.com.es/

Oficina de Naciones Unidas para la Reducción del Riesgo de Desastres (UNISDR): https://www.eird.org/americas/

Organización Panamericana de la Salud (OPS): hitps://www.paho.org/

Organización Mundial de la Salud (OMS): hHtps://www.who.int/es

OUR WORLD IN DATA: https://ourworldindata.org/water-access

Secretaría de Planificación y Programación de la Presidencia (SeGePLAN): hitps:// www.segeplan.gob.gt/nportal/

Sistema Nacional para la Prevención, Mitigación y Atención a Desastres (SINAPRED): http://www.sinapred.gob.ni/

Song, X., Zhang, Q., Sekimoto, Y., Shibasaki, R., Jing Yuan, N. y Xie, X. (2016). "Prediction and simulation of human mobility following natural disasters". ACM Transactions on Intelligent Systems and Technology, 29. DOI: 10.1145/2970819 
UNICEF: hHtps://www.unicef.es/

United States Census Bureau: hitps://www.census.gov/

Willamson, R. A., Hertzfeld, H. R., Cordes, J. y Logsdon, J. M. (2002). "The socioeconomic benefits of Earth science and applications research: reducing the risks and costs of natural disasters in the USA", Space Policy, 18, 57-65.

YANG, C., YANG, J., LUo, X. y GONG, P. (2009). “Use of mobile phones in an emergency reporting system for infectious disease surveillance after the Sichuan earthquake in China". Bulll World Health Organ, 87, 619-623. DOI: 10.2471/BLT.08.060905

Zorn, S., Rose, R., Goetz, A. y Weigel, R. (2010). "A novel technique for mobile phone localization for search and rescue applications". In: Proceedings of International Conference on indoor positioning and indoor navigation. Suiza: Zürich. 